\title{
Transiciones térmicas en recubrimientos polisiloxánicos de fibras de vidrio
}

\author{
J. GONZÁLEZ-BENITO ${ }^{1}$, A.J. AZNAR ${ }^{1}$, A. MAÇANITA ${ }^{2}$, J. BASELGA ${ }^{2}$ \\ ${ }^{1}$ Dpto. Ciencia de Materiales, Univ. Carlos III de Madrid, Leganés (SPAIN). \\ 2I.T.Q.B., Av. Rovisco Pais, Oeiras 1096, (Portugal).
}

\begin{abstract}
Se realizó una estimación de las temperaturas de relajación de recubrimientos poliméricos en fibras de vidrio. Para ello, se llevó a cabo el seguimiento de la respuesta fluorescente, en función de la temperatura, de dos fluoróforos químicamente enlazados al recubrimiento polimérico. Las fibras de vidrio fueron tratadas con una disolución acuosa de 3-aminopropiltrietoxisilano (APES), y una mezcla al $50 \%$ de APES y de 3-aminopropilmetildietoxisilano (APDES). Los marcadores fluorescentes, cloruro de pireno-1-sulfonilo (PSC) y cloruro de 1-dimetilamino-5-naftalenosulfonilo (DNS), se anclaron a las fibras de vidrio silanizadas a través de la formación de un compuesto sulfonamida estable y fluorescente (PSA y DNSA respectivamente). Se realizaron sus espectros de emisión en función de la temperatura en el intervalo (133, 413)K. Los resultados mostraron transiciones térmicas a bajas temperaturas para las muestras estudiadas indicando la alta flexibilidad de la región de acoplamiento. Además, dicha temperatura de transición depende de forma clara de la estructura del polímero que recubre a las fibras.
\end{abstract}

Palabras clave: Fibras de vidrio, 3-aminopropiltrietoxisilano, región de acoplamiento, marcadores fluorescentes.

\section{Thermal Transitions of Polysiloxane Coatings onto Glass fibers}

It was estimated the relaxation temperatures of polymeric coatings on glass fibers. In order to do this, it was carried out the fluorescent response study, as a function of temperature, of two dyes chemically bonded to the polymeric coating. The glass fibers were treated with aqueous solutions of 3-aminopropyltriethoxisilane (APES) and a $50 \%$ mixture of APES and 3-aminopropylmethyldiethoxisilane (APDES). The fluorescent labels, pyrene-1-sulfonyl chloride (PSC) and 1-dimethylamino-5naphtalenesulfonyl chloride (DNS), were attached to the silanized glass fibers by the formation of a stable and highly fluorescent sulfonamide conjugate (PSA and DNSA respectively). Emission spectra were recorded as a function of temperature in the range $(133,413) \mathrm{K}$. The results shown thermal transitions at low temperatures, which reflect the high flexibility of the coupling region. In addition, that transition temperature clearly depends on the structure of the polymeric coating of the glass fibers.

Key words: Glass fibers, 3-aminopropyltriethoxisilane, coupling region, fluorescent labels.

\section{INTRODUCCIÓN}

Los trialcoxisilanos se utilizan frecuentemente como agentes de acoplamiento para fibras de vidrio en materiales compuestos de matriz polimérica. Su presencia mejora la adhesión interfacial entre las fibras reforzantes y la matriz (1-3).

Los agentes de acoplamiento en base silano suelen reaccionar mediante condensación con los grupos silanoles superficiales del vidrio formando enlaces siloxánicos $\mathrm{Si}$ (sustrato)-OSi(silano). Debido a la geometría molecular de los silanos y al escaso número de silanoles en ciertas superficies silícicas, no es posible la condensación completa con el sustrato y, en su lugar, se produce homocondensación formándose una capa heterogénea de poliorganosiloxano que recubre, con mayor o menor eficacia al vidrio (4). Esta capa recibe el nombre de región de acoplamiento.

El mecanismo por el que la matriz interacciona con el recubrimiento depende en buena parte de la microestructura de esta región $(1,5)$. Las cadenas de polímero de la matriz deben difundir hacia la interfase y penetrar en ella para que la reacción de acoplamiento con la matriz pueda tener lugar. El acoplamiento químico en la interfase, la apertura de red y, sobre todo, su flexibilidad son por tanto factores decisivos para que la región de acoplamiento pueda soportar y/o relajar tensiones cortantes interfaciales en el material compuesto (6). Aunque es abundante la literatura científica sobre tipos de silanos y optimización de formulaciones de acoplamiento, no se conocen las propiedades de tales interfases. La mayor parte de los estudios realizados en este campo, basados en la variación de las propiedades mecánicas de los materiales compuestos fibra de vidrio/matriz polimérica, han justificado sus resultados en términos de una mayor o menor accesibilidad por parte de la matriz polimérica a la funcionalidad del silano (5), por ejemplo una mayor penetración del polímero en la interfase debe dar lugar a un aumento a la tensión interfacial (5). En cambio, poco se conoce del efecto que produce la variación de la flexibilidad de la región de acoplamiento en dichas propiedades mecánicas. Con objeto de poder correlacionar dichas propiedades, es necesario previamente conocer la rigidez de la región de acoplamiento, aspecto que hasta el momento no está del todo subsanado. 
El objetivo del presente trabajo consiste en estudiar las propiedades térmicas de un poliorganosiloxano quimisorbido a superficies de fibra de vidrio E.

La medida de la temperatura de transición vítrea del polímero de la región de acoplamiento puede proporcionar información muy útil para conocer la flexibilidad conformacional de las interfases en materiales compuestos. Desafortunadamente, la fracción en masa de polímero quimisorbido en las fibras es muy pequeña $(0,5 \%)$ y los métodos térmicos habituales no son adecuados. En este trabajo se emplea la técnica de marcadores fluorescentes para estudiar este tipo de transiciones en un intervalo amplio de temperaturas. El empleo de sondas y marcadores fluorescentes ha supuesto un gran avance en el conocimiento, a escala molecular, de sistemas heterogéneos como los biológicos, superficies modificadas (7-9), etc. Sus principales ventajas son su alta sensibilidad y el bajo grado de perturbación sufrido por el sistema en estudio. Los parámetros fotofísicos de algunos compuestos luminiscentes dependen de manera directa de la polaridad $(7,10)$ o la rigidez del microentorno $(7,11)$, de manera que sus valores pueden ofrecer una escala numérica que describa el progresivo cambio de entorno polar a no polar, en un caso, y de entorno rígido a flexible en otro. El análisis de la fluorescencia en las proximidades de una transición térmica que implique cambios en la movilidad molecular del marcador nos permite detectar su presencia.

\section{PROCEDIMIENTO EXPERIMENTAL}

\subsection{Materiales}

Fibras de vidrio E suministradas por VETROTEX. Se emplearon como agentes de acoplamiento para recubrir fibras de vidrio dos aminosilanos suministrados por ABCR: 3-aminopropiltrietoxisilano (APES), 3-aminopropilmetildietoxisilano (APDES) sin purificación previa. Los marcadores fluorescentes utilizados fueron cloruro de pireno-1-sulfonilo (PSC) suministrado por Molecular Probes y cloruro de 1-dimetilamino-5-naftalenosulfonilo (DNS) suministrado por ICN Pharmaceuticals. Por último, todos los disolventes empleados, suministrados por Aldrich, fueron de calidad HPLC o superior. En todos los casos se emplearon tal y como fueron recibidos sin posteriores purificaciones excepto en el caso del tolueno que fue destilado sobre sodio para eliminar agua.

\subsection{Preparación de muestras}

\subsubsection{PRETRATAMIENTO DE FIBRAS}

Las fibras de vidrio comerciales, antes de ser utilizadas, fueron calcinadas en un horno a $450^{\circ} \mathrm{C}$ durante $1 \mathrm{~h}$. Esta temperatura de calcinación se ha seleccionado de forma que, por una parte, sea lo suficientemente alta para que la oxidación de la materia orgánica sea completa y, por otra, lo suficientemente baja como para evitar una deshidroxilación excesiva de la superficie (12). Después de la calcinación, las fibras de vidrio fueron sometidas a un tratamiento de activación mediante un reflujo de $\mathrm{HCl} 10 \%$ durante 3 h con el fin de regenerar los grupos silanol superficiales.

\subsubsection{SILANIZACIÓN}

Se introdujo 1 gramo de fibras de vidrio activadas en $50 \mathrm{ml}$ de disolución acuosa al 1\% (v/v) de: i) APES puro (muestra A)

ii) Mezcla APES + APDES; 50\% APES (muestra B) a temperatura ambiente durante $10 \mathrm{~min}$. Después del correspondiente proceso de silanización, las fibras de vidrio fueron sometidas a un proceso de calentamiento durante una hora, para conseguir la policondensación de los residuos etoxi además de un secado. Posteriormente, dichas fibras secas fueron sometidas a un proceso de extracción en soxhlet con tolueno seco con el fin de eliminar todo el silano que no hubiera reaccionado. Por último, se procedió a un secado a vacío a temperatura ambiente durante al menos $8 \mathrm{~h}$.

\subsubsection{DANSILACIÓN Y PIRENACIÓN}

Las fibras de vidrio silanizadas fueron marcadas con cloruro de dansilo (DNS) o cloruro de pirenosulfonilo (PSC). Para ello, el cromóforo DNS o PSC fue anclado a las fibras vía la formación de una sulfonamida (DNSA y PSA respectivamente) con las funcionalidades amina procedentes del recubrimiento poliorganosiloxánico. El tratamiento consistió en sumergir $0.4 \mathrm{~g}$ de fibras silanizadas en $25 \mathrm{ml}$ de una disolución de marcador $\left(10^{-4} \mathrm{M}\right)$ en acetonitrilo durante $10 \mathrm{~min}$. a temperatura ambiente, proceso de extracción en soxhlet con tolueno seco para eliminar cromóforo absorbido y secado a vacío durante al menos $8 \mathrm{~h}$ a temperatura ambiente.

\subsubsection{PREPARACIÓN DE UN POLÍMERO MODELO}

Una disolución acuosa de APES al $10 \%$ v/v se mantuvo a temperatura ambiente con objeto de evaporar toda el agua. Posteriormente, el polímero obtenido se mantuvo a vacío a $60{ }^{\circ} \mathrm{C}$ durante $24 \mathrm{~h}$ con objeto de secarlo y obtener un alto grado de polimerización.

\subsection{Técnicas instrumentales}

Los espectros de fluorescencia fueron realizados en un fluorímetro SPEX con doble monocromador tanto en excitación como en emisión, con el que se registraron los espectros a la velocidad de $120 \mathrm{~nm} / \mathrm{min}$ y se utilizaron rendijas tanto de excitación como de emisión de anchura $1 \mathrm{~nm}$. Las medidas se realizaron mediante excitación frontal, introduciendo las fibras de vidrio en un dispositivo diseñado para tal fin. Este dispositivo consta de un soporte plano (donde se colocan las fibras que quedan fijas al encajar una ventana de cuarzo) unido por un tornillo a un cilindro graduado de manera que, el simple giro de dicho tornillo permita en todo momento seleccionar el ángulo de incidencia de la radiación. Para controlar temperatura sobre la muestra, la célula se coloca en un portacélulas con camisa de refrigeración.

Los termogramas fueron realizados a una velocidad de calentamiento de $10{ }^{\circ} \mathrm{C} / \mathrm{min}$ en un calorímetro DSC-7 de Perkin Elmer.

\section{RESULTADOS Y DISCUSIÓN}

Se estudió la variación con la temperatura de la emisión fluorescente del grupo DNSA y PSA cuando se encuentran anclados a fibras de vidrio silanizadas. Con el fin de analizar el efecto de la estructura del polímero se han utilizado 2 tipos de recubrimientos diferentes en las fibras, muestras A y B. Es de esperar que la adición de un monómero difuncional a un trifuncional modifique sustancialmente el grado de entrecruzamiento del polímero. 


\subsection{PSA}

En la figura 1 se muestran espectros de fluorescencia de PSA anclado a fibras de vidrio silanizadas (muestra A) a varias temperaturas. En todos los casos se observan dos regiones características en estos sistemas (8,9): i) de 350 a 420 nm, emisión monomérica y ii) de 420 a 540 nm, emisión excimérica procedente de exciplejos formados a partir de la interacción entre un grupo PSA excitado (aceptor) y un grupo amino sin reaccionar del polisiloxano. Se puede observar que ambas emisiones disminuyen al aumentar la temperatura con algunas discontinuidades que serán analizadas más tarde, y que los máximos de emisión de monómero se desplazan al rojo con la temperatura.

En la figura 2(a), se representa la longitud de onda del máximo de emisión correspondiente a la transición 0-0 $\left(\lambda_{0-0}\right)$ en función de la inversa de la temperatura.

Los desplazamientos espectrales dependientes de la temperatura para procesos de absorción y emisión de moléculas apolares en disolventes apolares (como por ejemplo PSA en polisiloxano), normalmente se interpretan teniendo en cuenta el balance entre dos efectos opuestos (13): i) al aumentar la temperatura, la población de ciertos niveles vibracionales puede perturbarse, cambiando la forma de la banda de transición y desplazándola a bajas energías; ii) y al mismo tiempo, la densidad del disolvente puede disminuir, dando lugar a un desplazamiento a mayores energías debido a que el efecto de estabilización por fuerzas de dispersión (dipolo inducido - dipolo inducido) también disminuye.

En el caso que nos ocupa, según la figura 2(a) al aumentar la temperatura desde $133 \mathrm{~K}$ hasta $150 \mathrm{~K}$, se observa un ligero desplazamiento al rojo (batocromismo) que se interpreta como un aumento en la población de los niveles vibracionales. Desde $150 \mathrm{~K}$ este hábito batocrómico se hace más lento hasta $180 \mathrm{~K}$, momento a partir del cual se detiene hasta $210 \mathrm{~K}$. De acuerdo con la interpretación clásica mencionada anteriormente, estos cambios sólo se pueden explicar porque en estos intervalos de temperaturas se percibe el efecto de la disminución de densidad del entorno de la pirensulfonami$\mathrm{da}$, siendo el efecto predominante en el intervalo $(180,210) \mathrm{K}$. A partir de $210 \mathrm{~K}$ el hábito batocrómico se vuelve a recuperar.

En la figura 2(b) se presenta la representación de Arrhenius correspondiente a la relación de intensidades de fluorescencia a $470 \mathrm{~nm}$ y a $397 \mathrm{~nm}\left(\mathrm{I}_{\mathrm{E}} / \mathrm{I}_{\mathrm{M}}\right)$. Se puede observar que, al calentar desde $133 \mathrm{~K}$ nos encontramos con dos máximos en la relación $\mathrm{I}_{\mathrm{E}} / \mathrm{I}_{\mathrm{M}}$. Un máximo en esta relación se puede interpretar como aquella temperatura a la cual la formación de exciplejo deja de estar controlada por difusión para estar controlada por la entalpía de reacción. Teniendo en cuenta que la región de acoplamiento correspondiente a este tipo de recubrimientos parece estar compuesta por dos regiones, una en la que los grupos amino presentan mayor movilidad que en la otra $(9,14)$, el máximo encontrado a menores temperaturas $(180 \mathrm{~K})$ se debe corresponder con el control por reacción de exciplejos formados con moléculas de PSA en entornos flexibles, mientras que el máximo a mayores temperaturas $(285 \mathrm{~K})$ de exciplejos formados con moléculas de PSA en entornos más rígidos.

Sin embargo, la energía de activación de exciplejo en la región de bajas temperaturas $(\mathrm{T}<180 \mathrm{~K})$ que se obtiene de la pendiente tiene un valor extremadamente bajo $(\sim 0.1 \mathrm{kcal} /$ $\mathrm{mol})$, bastante inferior a las barreras rotacionales de enlaces

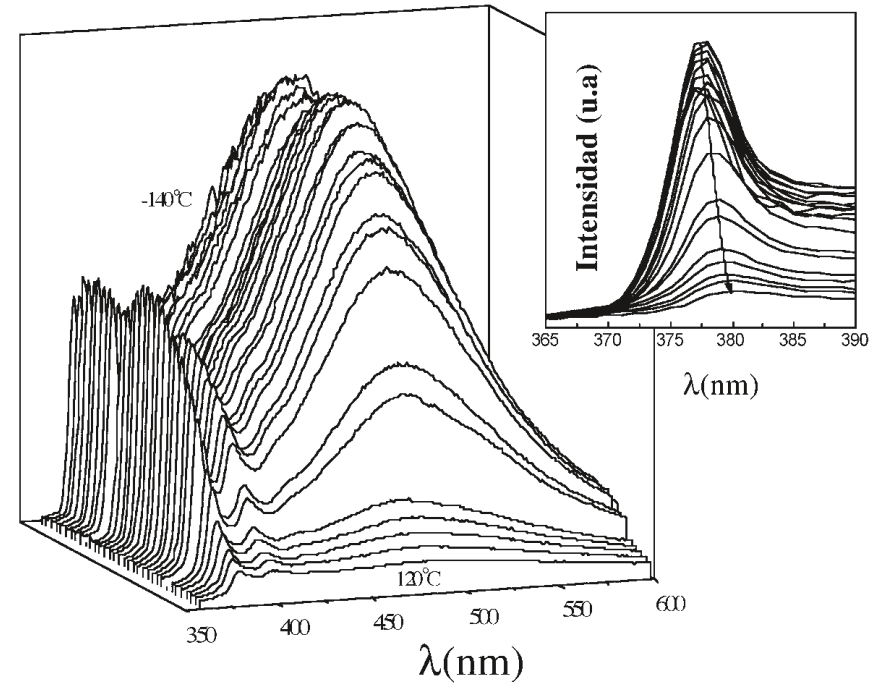

Figura 1.- Espectros de fluorescencia de PSA anclado a fibras de vidrio silanizadas (muestra $\mathrm{A}$ ) a varias temperaturas.

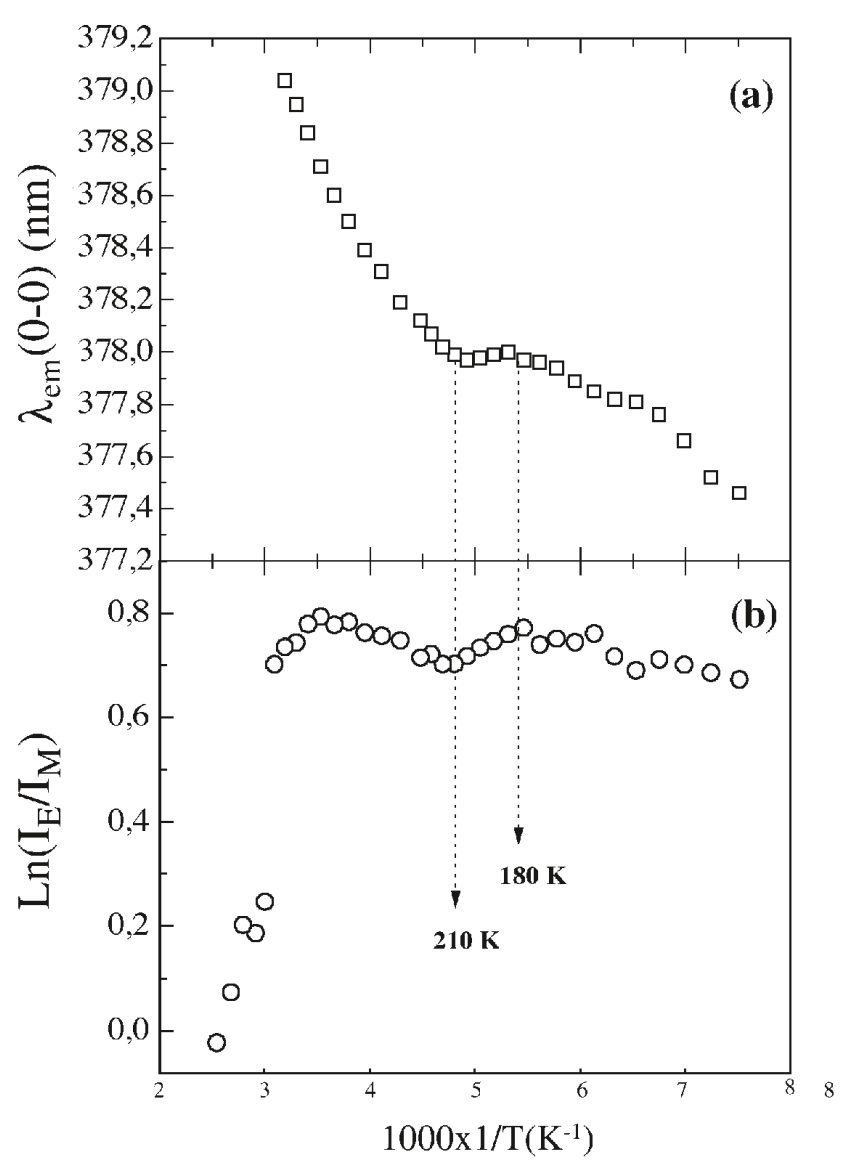

Figura 2.- (a) Variación de la longitud de onda del máximo de emisión de la transición 0-0 del PSA (muestra A) en función de la inversa de la temperatura y (b) curva de Arrhenius para la relación de intensidades exciplejo/monómero. 
típicos, incluso en siloxanos. El tipo de movimientos que se activan en esta región de temperaturas no deben ser rotaciones completas sino más bien ligeros cambios en la orientación del cromóforo respecto de la amina.

A la vista de este análisis se podría concluir que los resultados obtenidos sugieren la detección de importantes relajaciones de la matriz asociadas a variaciones en la densidad dentro del intervalo de temperaturas estudiado; es decir, la emisión de PSA anclado a fibras de vidrio silanizadas es capaz de detectar transiciones térmicas del polímero (polisiloxano) que recubre a las fibras.

\subsection{DNSA}

Para estudiar el efecto de la temperatura en la emisión del grupo DNSA se han escogido dos parámetros fotofísicos: intensidad del máximo de fluorescencia (I) y energía de dicho máximo $\left(v_{\mathrm{e}}\right)$.

El DNSA anclado a fibras de vidrio silanizadas presenta una variación muy peculiar en el rendimiento cuántico de la fluorescencia (expresado como variación del logaritmo neperiano de la intensidad de emisión) en función de la temperatura. Esta variación está representada en la figura 3 para las muestras A y B donde se puede observar el siguiente comportamiento:

A bajas temperaturas, desde $133 \mathrm{~K}$, hay una disminución progresiva en la intensidad de emisión a medida que la temperatura aumenta hasta $180 \mathrm{~K}$ en las fibras A y hasta $195 \mathrm{~K}$ en las fibras B. El posterior aumento de la temperatura provoca un incremento de la intensidad hasta llegar a una temepratura de $208 \mathrm{~K}$ en las fibras A y hasta $238 \mathrm{~K}$ en las B. A partir de estas temperaturas, la intensidad de fluorescencia vuelve a disminuir tendiendo a cero a altas temperaturas.

Estos resultados se pueden explicar teniendo en cuenta que el dansilo se caracteriza por poseer dos estados excitados próximos en energía (15), con dos conformaciones diferentes y, por tanto, rendimientos cuánticos distintos. Según lo anterior, es previsible que a bajas temperaturas sólo puedan existir grupos DNSA con la conformación más estable. $\mathrm{Al}$ aumentar la temperatura, la intensidad de fluorescencia total debería disminuir, tal y como se observa en la figura 3, porque los procesos de desexcitación no radiativos aumentan. Esta disminución ocurre hasta una temperatura que llamaremos $\mathrm{T}_{1}$ a partir de la cual la intensidad de fluorescencia aumenta. Este aumento de intensidad podría ser debido a que se ha aportado la suficiente energía como para permitir, en el estado excitado, un cambio en la conformación del grupo DNSA. Si el rendimiento cuántico de la fluorescencia asociado a esta conformación es suficientemente mayor que el asociado a la más estable como para compensar el efecto debido al aumento del decaimiento no radiativo, quedaría justificado el aumento de intensidad.

Posteriormente, tal y como queda reflejado en la figura 3, la intensidad de fluorescencia vuelve a disminuir porque el aumento en el decaimiento no radiativo es lo suficientemente grande como para que la fluorescencia total disminuya.

Ahora cabría preguntarse si los resultados obtenidos se deben a una característica intrínseca del fluoróforo o a un efecto inducido por el medio que lo rodea. La comparación entre las dos curvas de la figura 3 refleja que las fibras A presentan un mínimo $\mathrm{T}_{1}=180 \mathrm{~K}$ inferior al de las fibras $\mathrm{B}$ en las que $T_{1}=195 \mathrm{~K}$. Esta diferencia sugiere que la estructura de la matriz es la que condiciona el cambio de conformación en el estado excitado del DNSA, es decir, la mayor o menor rigidez del entorno próximo al marcador.

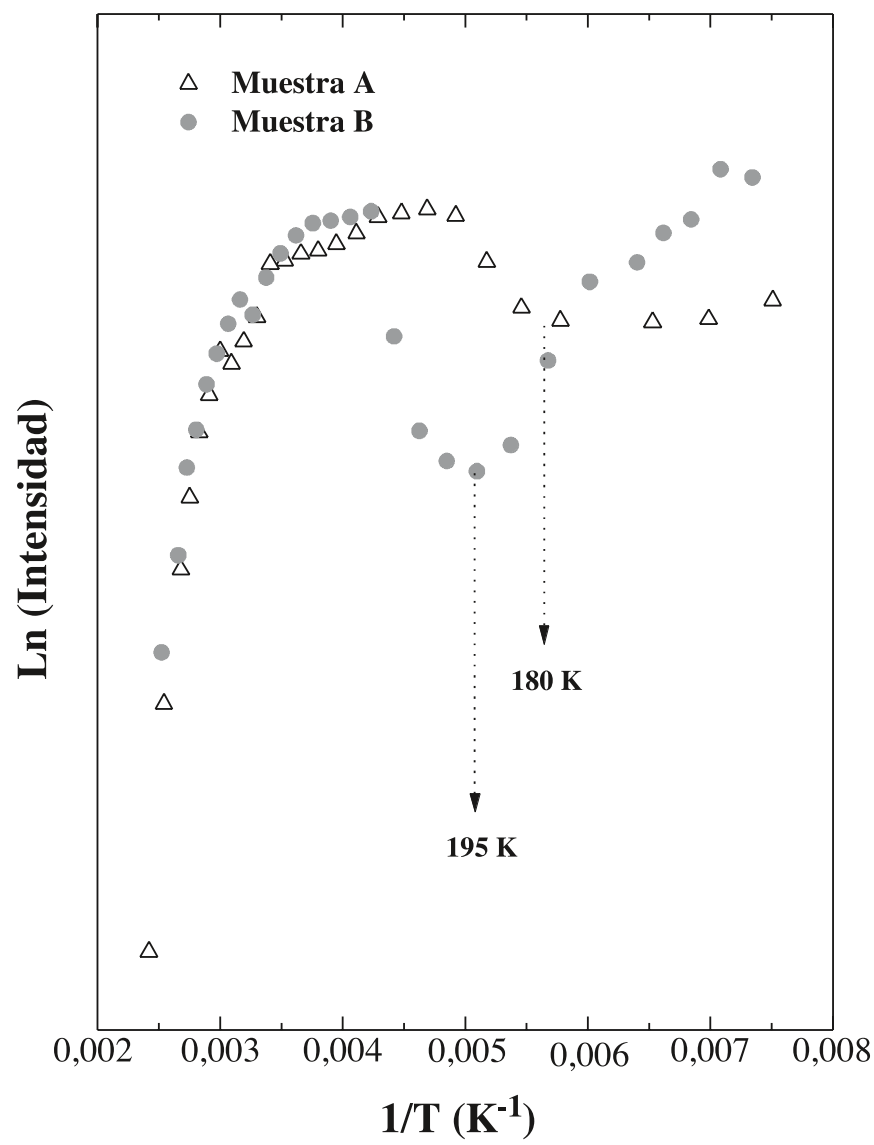

Figura 3.- Variación con la temperatura de la intensidad del máximo de fluorescencia del grupo dansilo anclado a fibras de vidrio silanizadas.

Según lo anterior, se podría concluir entonces que la capa poliorganosiloxánica que recubre a las fibras A debe caracterizarse por poseer menor impedimento en los movimientos locales de cadena, es decir, su estructura debe tener menor rigidez o el polímero ser más abierto.

La estabilización del estado excitado de un cromóforo puede venir dada por la reorientación de dipolos de las moléculas de disolvente (relajación) en el que se encuentra inmerso, siempre que el estado excitado posea un momento dipolar considerable (16). Pero este proceso dependerá del tiempo que necesiten dichas moléculas para reorientarse en relación con el tiempo de vida del estado excitado y, por tanto, de su movilidad. Como la movilidad de las moléculas de un sistema es función de la temperatura, parece evidente que la temperatura va a jugar un papel primordial a la hora de producirse la estabilización del estado excitado del DNSA. Por consiguiente, la cinética de relajación del disolvente se puede analizar mediante la dependencia de los espectros de emisión fluorescente con la temperatura, pudiéndose obtener información, en el caso de considerar como disolvente una matriz polimérica, de sus transiciones térmicas.

La energía correspondiente a la emisión del marcador DNSA permanece prácticamente constante e igual para las dos muestras estudiadas por debajo de una temperatura de transición que se aproxima a la de los mínimos en las curvas 
representadas en la figura $3, \mathrm{~T}_{1}$. Este resultado podría explicarse considerando que, a muy bajas temperaturas, el estado electrónico del DNSA sólo se estabiliza por reorientación electrónica (16), estabilización que es tan pequeña que no se puede observar en los espectros de emisión; es decir, la reorientación de los dipolos está impedida debido a la rigidez conformacional de la matriz. Por encima de la temperatura de transición aparecen otras contribuciones a la estabilización del estado excitado, como una reorientación dipolar más rápida y la formación de un nuevo estado excitado del DNSA con conformación distinta que posee un mayor momento dipolar y por tanto capaz de dar lugar a un acoplamiento dipolar más efectivo con la matriz.

El valor del número de ondas correspondiente al máximo de emisión en la muestra A siempre es menor que en la muestra B cuando la temperatura es inferior a $315 \mathrm{~K}$ aproximadamente. Sin embargo, a altas temperaturas los máximos de emisión coinciden, lo cual sugiere que, a altas temperaturas, tanto las fibras A como las B ofrecen entornos similares al marcador.

Para las muestras estudiadas (figura 4) se han obtenido dos temperaturas de transición distintas, $155 \mathrm{~K}$ para la muestra A y $205 \mathrm{~K}$ para la muestra B, ambas temperaturas de transición son relativamente bajas para polímeros, indicándonos que en estos recubrimientos el grado de entrecruzamiento no debe ser muy alto. Esta conclusión estaría de acuerdo con el bajo valor de la temperatura de transición vítrea obtenida para el poliaminosiloxano (polímero modelo) utilizado, que fue de $223 \mathrm{~K}$. Por otro lado, si comparamos los resultados de las dos muestras entre sí encontramos que $\mathrm{T}_{1}$ (muestra $\mathrm{A}$ ) $<\mathrm{T}_{1}$ (muestra $\mathrm{B}$ ). Este resultado parece contradecir el hecho de que mayor grado de entrecruzamiento debería dar lugar a mayor rigidez. Una posible explicación podría ser, que mayor grado de entrecruzamiento no implica mayor rigidez, sino solamente mayor cantidad de agente de acoplamiento, dando lugar a una estructura más abierta.

\section{CONCLUSIONES}

Se estudiaron las propiedades térmicas de un poliorganosiloxano quimisorbido a superficies de fibra de vidrio E con objeto de conocer la rigidez de la región de acoplamiento. Para ello se ha analizado el efecto de la temperatura en algunos parámetros fotofísicos de dos moléculas fluorescentes ancladas a dicho polisiloxano. Se ha demostrado que la fluorescencia es lo suficientemente sensible como para estimar transiciones térmicas de la región de acoplamiento y de esta manera tener una idea de su flexibilidad. Por último, la comparación entre dos recubrimientos obtenidos a partir de: i) un aminosilano trifuncional (muestra A) y una mezcla entre un tri y un difuncional al 50\% (muestra B), refleja que el último, aun suponiendo menor grado de entrecruzamiento, tiene mayor rigidez, al menos, a escala molecular en el entorno del marcador fluorescente.

\section{BIBLIOGRAFÍA}

1. Plueddemann, "Silane Coupling Agents", Plenum Press, New York, (1982).

2. K.L. Mittal, "Silanes and Other Coupling Agents", JSP, Ed. Utrech, (1992).

3. N. Suzuki, H. Ishida, "A Review on the Structure and Characterization Techniques of Silane/Matrix Interphases", Macromol. Symp., 108, 19-53, (1996).

4. E.T. Vandenberg, L. Bertilsson, B. Liedberg, K. Uvdal, R. Erlandsson, H. Elwing, I. Lundström, "Structure of 3-Aminopropyl Triethoxy Silane on Silicon Oxide", J. Colloid. Inter. Sci., 147, 1, 103-118, (1991).

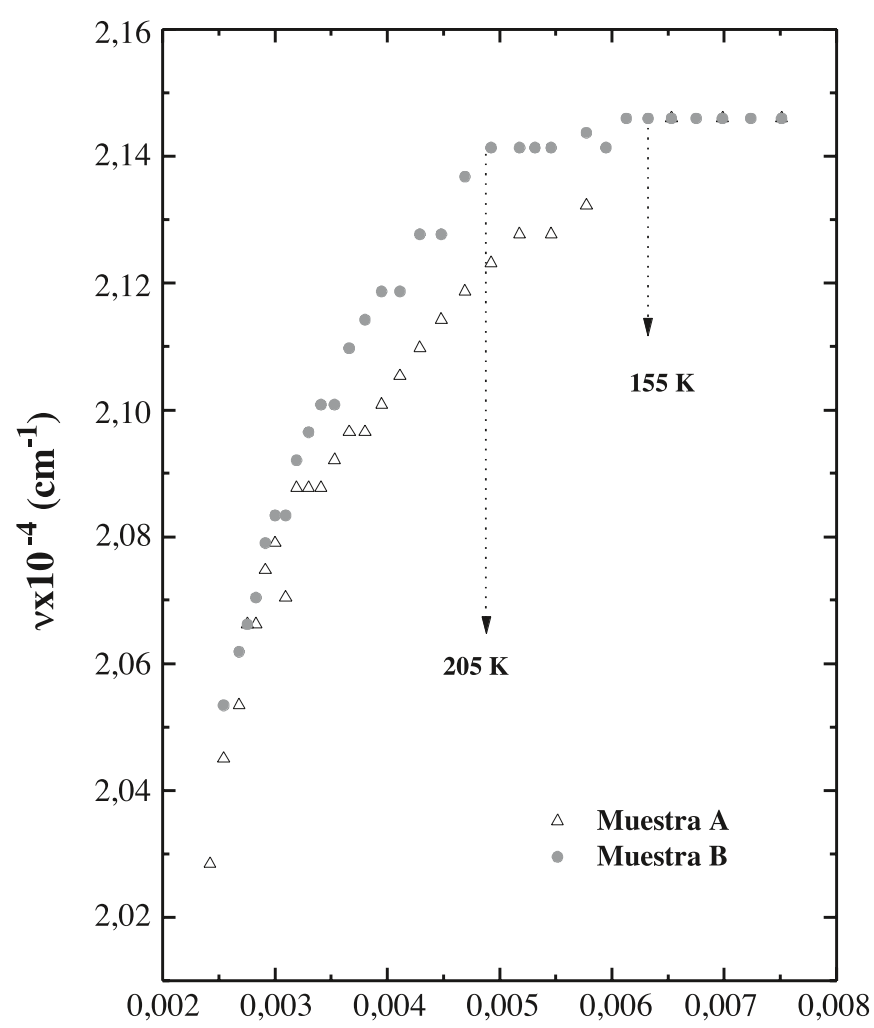

$\mathbf{T}\left(\mathbf{K}^{\mathbf{1}}\right)$

Figura 4.- Variación del número de ondas (máximo de emisión del DNSA anclado a fibras de vidrio silanizadas) con la inversa de la temperatura.

5. H. Hamada, N. Ikuta, N. Nishida, Z. Maekawa, “Effect of Interfacial silane network structure on interfacial strength in glass fibre composites", Composites, 25, 7, 512-515, (1994).

6. B.Z. Jang, "Advanced Polymer Composites: principles and applications", ASM International, (1994).

7. J. González-Benito, J.C. Cabanelas, A.J. Aznar, M.R. Vigil, J. Bravo, J. Baselga, "Surface Characterization of Silanized Glass Fibers by Labeling with Environmental Sensitive Fluorophores", J. Applied Polym. Sci., 62, 375-384, (1996).

8. J. González-Benito, J.C. Cabanelas, A.J. Aznar, M.R. Vigil, J. Bravo, B. Serrano, J. Baselga, "Photophysics of a Pyrene Probe Grafted onto Silanized Glass Fiber Surfaces", J. Luminiscence, 72-74, 451-453, (1997).

9. J. González-Benito, J.C. Cabanelas, A.J. Aznar, M.R. Vigil, J. Bravo, J. Baselga, "Pyrene-Doped Polyorganosiloxane Layers over Commercial Glass Fibers", J. Fluorescence, 9, 1, 51-57, (1999).

10. P. Suppan, "Invited Review Solvatochromic Shifts: The Influence of the Medium on the Energy of Electronic States", J. Photochem. Photobiol. A: Chem., 50, 293-330, (1990).

11. K. Ficht, K. Fisher, H. Hoff, C.D. Eisenbach, “Fluorescent Probe Studies of Polymer-Solvent Interactions", Makromol. Chem. Rapid Commun., 14, 515-521, (1993).

12. J. González-Benito, "Modificación Superficial de Fibras de Vidrio. Microestructura, Propiedades y Degradación", Tesis Doctoral, Dpto. Química Física I, U.C.M, (1999).

13. R.S. Becker, "Theory and Inerpretation of Fluorescence and Phosphorescence", Wiley Interscien, New York, (1980).

14. C.W. Chu, D.P. Kirby, P.D. Murphy, "Interactions of Aminosilane with Alumina and Silica Substrates Deposited from nonaqueous and aqueous media", J. Adhesion Sci. Technol., 7, 5, 417-433, (1993).

15. Y. Li, L. Chan, L. Tyer, R.T. Moody, C.M. Himel, D.M. Hercules, "Study of Solvents Effects on the Fluorescence of 1-(Dimethylamino)-5naphtalenesulfonic Acid and Related Compounds", J. Am. Chem. Soc., 97, 11, 3118-3126, (1975).

16. J.R. Lakowicz, "Principles of Fluorescence Spectroscopy", Plenum Press, New York, (1986). 